\title{
Size Reduction of Cavity-Backed Slot Antennas
}

\author{
Wonbin Hong, Student Member, IEEE, Nader Behdad, Student Member, IEEE, and Kamal Sarabandi, Fellow, IEEE
}

\begin{abstract}
A technique for reducing the dimensions of the cavity of a cavity-backed slot antenna (CBSA) is presented, which facilitates proper fabrication and integration of miniaturized slot antennas on multilayer substrates. This is accomplished by replacing the solid metal around the traditional slot antennas with a specific metallic pattern that can be viewed as a series of parallel strip lines placed around the slot antenna. This metallic pattern is then modified by designing the parallel strips in a compact fashion to reduce the overall antenna dimensions and obtain a reduced-size CBSA. It is shown that, for a simple straight slot antenna, the overall occupied volume of the modified cavity backing the slot antenna can be reduced by more than $65 \%$ without affecting the high radiation efficiency of the antenna. A number of traditional cavity backed slot antennas and the proposed modified CBSAs are designed, fabricated, and measured. The reduced-size CBSAs show a very low input VSWR, low cross-polarized radiation levels, and high radiation efficiency. Despite their small ground plane size, the proposed cavity backed slot antennas have front-to-back ratio (FTBR) values in the range of $6-7 \mathrm{~dB}$.
\end{abstract}

Index Terms-Aperture antennas, cavity-backed antennas, microstrip antennas, slot antennas.

\section{INTRODUCTION}

W ITH the current advancements in communication technology and tremendous growth in the wireless communication market and consumer demands, the need for smaller, more reliable, and power-efficient integrated wireless systems is more than ever felt. For future wireless devices, implementation of the entire transceivers on a single chip is being envisioned with the goal of reducing the cost of such systems and making them more affordable. At low microwave frequencies, where signal propagation through man made obstacles is possible to some extent, antennas appear to be the largest components of any such system. Antenna miniaturization is then an important task toward achieving the integration and miniaturization of wireless communication systems. In a recent study, it is shown that miniaturized slot antennas have higher bandwidth and efficiency compared to electrically small wire type antennas [1] and a number of different techniques for designing highly efficient miniaturized slot antennas have been reported in [1]-[4]. One drawback of slot antennas, however, is their inherent bidirectional radiation. In many applications, the antenna needs to be located in close proximity to earth or conductive bodies, be mounted on a platform, or be integrated with the rest of the transceiver in a multilayer structure. To alleviate the adverse effects of the interactions between a slot antenna and the structures behind it, traditionally a shallow cavity is placed behind such an antenna.

Manuscript received August 26, 2005; revised December 7, 2005.

The authors are with the Radiation Laboratory, Department of Electrical Engineering and Computer Science, The University of Michigan, Ann Arbor, MI 48109 USA (e-mail: saraband@umich.edu).

Digital Object Identifier 10.1109/TAP.2006.874351
Cavity-backed slot antennas (CBSAs) have extensively been studied both analytically and experimentally and are among the most basic types of aperture antennas [5]. The admittance of a rectangular-slot antenna, backed by a rectangular cavity has analytically been derived in [6] and experimentally verified in [7]. In [8], the admittance of a CBSA is mathematically related to the admittance of an ordinary slot antenna and the results are later experimentally verified in [9]. Aperture field and circuit parameters of CBS antennas have also been investigated and the results are presented in [10]. Size reduction of the CBSA is achieved through dielectric and ferrite loading in the cavity and the experimental results are compared to its theoretical values [11]. In addition to its basic form, variations of the ordinary CBSAs have also been used for a number of different applications. In particular, an active CBSA is presented in [12] and a circularly polarized CBSA is studied in [13]. In [14], a wide-slot antenna backed by a cavity is used to increase the bandwidth of, otherwise narrowband, CBSAs. In another attempt to increase the bandwidth and directivity of the naturally narrowband CBSAs, a log-periodic arrangement is considered in [15] to achieve a wideband end-fire CBSA that has a fractional impedance bandwidth greater than $64 \%$. Feed modification is performed in [16] by using a T-bar shape feed to enhance the bandwidth and improve the relative gain.

In this paper, we present a method for reducing the cavity dimensions of a slot antenna backed by a rectangular cavity and examine the performance of such antennas. The technique is based on substituting the cavity structure with a series of miniaturized transmission line type resonators. Basically the slot antenna is initially designed using a finite width metallic strip connected to a number of closely spaced parallel short-circuited microstrip lines with the same physical and electrical length as the width of the ground plane. These parallel lines are then meandered to reduce their physical length, while maintaining their electrical length, thereby resulting in an overall reduction in the physical dimensions of the CBSA. In what follows, first the method for modifying the structure of an ordinary CBSA is presented in Section II and then the cavity miniaturization technique is presented in Section III. In both sections, simulation and measurement results of the antennas are presented and discussed.

\section{CBSAs With ModifiEd GROUND Planes}

A slot antenna is usually viewed as an equivalent resonant magnetic dipole with its first resonance occurring at a frequency where it has an overall length of $\lambda / 2$. A major drawback of slot antennas is their inherent bidirectional radiation, which not only directs half of the power in potentially undesired directions but also increases the sensitivity of the antenna to its surrounding environment and prohibits the placement of such an- 
tennas on platforms or multilayer circuit boards. For example, placing such a slot antenna in close proximity of a large metallic object, such as a vehicle, can significantly change the input impedance and radiation patterns of the antenna. A common solution to circumvent this problem is to implement a lossy cavity behind the slot antenna in order to suppress the backward radiation and conserve the input impedance.

In this paper, we use an off-centered open-circuited microstrip line, with a line impedance of $50 \Omega$ to feed the cavity backed slot antenna. The slot and the microstrip line are printed on the two different sides of a thin dielectric substrate with relative dielectric constant of $\epsilon_{r a}$. This substrate is then placed on the cavity such that the slot is sandwiched between the bottom of the cavity and the microstrip line. The cavity itself is filled with a dielectric material with dielectric constant of $\epsilon_{r c}$. The length of the open-circuited microstrip line $L_{m}$ and its location $L_{f}$ can be tuned to match the antenna's input impedance to that of the microstrip feed. In order to have an efficient radiator, the cavity width must be chosen close to $\lambda_{c} / 2$ at the frequency of operation, where $\lambda_{c}=\lambda_{0} / \sqrt{\epsilon}_{r c}$ is the wavelength inside the cavity and $\lambda_{0}$ is the wavelength in free space. At this frequency, the slot antenna has an approximate length of $\lambda_{g} / 2$, where $\lambda_{g}$ is the guided wavelength along the slotline and in general it is different from $\lambda_{c}$. Such a CBSA is designed and simulated using a method of moments (MoM) based commercial simulation tool [20]. The rectangular slot has dimensions of $L_{s}=50 \mathrm{~mm}$ and $W_{s}=2 \mathrm{~mm}$ and is fabricated on a $0.5 \mathrm{~mm}$ thick RO4003 substrate from Rogers Corp. The substrate has a dielectric constant of $\epsilon_{r a}=3.4$, loss tangent of $\tan \delta_{a}=0.0027$, and overall dimensions of $L_{g}=53 \mathrm{~mm}$ and $W_{g}=46 \mathrm{~mm}$. The cavity is fabricated by machining a piece of $6.35 \mathrm{~mm}$ thick TMM3 substrate (from Rogers Corp.) and metallizing its sides. The material inside the cavity, TMM3, has a dielectric constant of $\epsilon_{r c}=3.3$ and loss tangent of $\tan \delta_{c}=0.0020$. The antenna is fed using an off-centered open-circuited microstrip line, with an impedance of $50 \Omega$, and it is matched to $50 \Omega$ by optimizing the length of the open circuited stub $L_{m}$ and the feed location, $L_{f}$, using full wave simulations. The complete geometrical dimensions and physical parameters of this antenna are listed in Table I.

The fabricated ordinary cavity backed slot antenna operates at $2.25 \mathrm{GHz}$ and there is a good agreement between the measured and simulated results. The radiation patterns of the antenna are measured in the E-plane ( $y-z$ plane $)$ and H-plane $(x-z$ plane $)$ inside the anechoic chamber of the University of Michigan. It is observed that the antenna has low levels of cross polarized radiation in both E- and H-planes and it has a front-to-back ratio (FTBR) of about $8.6 \mathrm{~dB}$. The FTBR, for fixed cavity dimensions, can be increased by increasing the dimensions of the antenna's ground plane. This, however, will increase the overall dimensions of the antenna, which may not be desirable in certain applications.

In order to evaluate the miniaturization potential of this cavity backed slot antenna, it is important to first study the flow of electric current in the ground plane around the slot antenna. The electric current can be decomposed into two components: one that circulates around the slot that is mainly responsible for creating a resonant condition and one that flows perpendicular to the slot.
TABLE I

Physical Dimensions of CBSAs of SECTION II. All Dimensions ARE IN mm

\begin{tabular}{|c||c|c|c|c|c|c|}
\hline Parameter & $L_{s}$ & $W_{s}$ & $L_{g}$ & $W_{g}$ & $L_{c}$ & $W_{c}$ \\
\hline Value & 50 & 2 & 53 & 46 & 53 & 46 \\
\hline Parameter & $L_{m}$ & $L_{f}$ & $h_{c}$ & $h_{a}$ & $\epsilon_{r c}$ & $\epsilon_{r a}$ \\
\hline Value & 18 & 2.4 & 6.35 & 0.5 & 3.3 & 3.4 \\
\hline
\end{tabular}

It is this latter component that is mainly responsible for radiation from the slot antenna. The electric current flowing parallel to the slot and above it has the same magnitude as the current that is flowing parallel to the slot and below it but these two components are $180^{\circ}$ out of phase and hence, they do not contribute to the far field radiation. As a first step toward reducing the dimensions of this antenna, we will replace the uniform metallic plane around the slot antenna with a loop defining the slot and a number of strips perpendicular to it as shown in Fig. 1. Here, the metallic loop around the slot supports the circulating component of the electric current and the parallel metallic strips support the normal component of the electric current. Since the parallel strips carry the radiating component of the electric current, their total number plays an important role in the radiation efficiency of the antenna. If the number of these strips is high enough, the response of the antenna will not be significantly different from the ordinary CBSA. However, decreasing the number of strips decreases the number of available paths for the normal component of the electric current and consequently decreases the density of the radiating current and hence decreases the radiation efficiency of the antenna. In this paper, we take an experimental approach to validate this hypothesis by designing and fabricating three CBSAs with modified geometry each having one, five, and 30 strips of equal widths. The differences between these three antennas are the number of strips, the length of the open circuited microstrip feed $L_{m}$, and the feed location $L_{f}$. The geometrical values of these parameters and antenna dimensions are presented in Table II and in Fig. 1.

The input reflection coefficient of these antennas are measured using a calibrated HP8720D vector network analyzer and the results are presented in Fig. 2. It is shown that, all three antennas can easily be matched to $50 \Omega$ by tuning the location and length of the open-circuited microstrip line $L_{m}$ and $L_{f}$. The difference in the resonant frequencies can be explained by the variations of $L_{m}$ and $L_{f}$ and deviations caused during the fabrication process. The far-field copolarized $\left(\left|E_{\theta}\right|^{2}\right)$ and cross-polarized $\left(\left|E_{\phi}\right|^{2}\right)$ radiation patterns of these antennas are measured in the E- and H-planes ( $y-z$ and $x-z$ planes) inside the anechoic chamber of the University of Michigan. Results are presented in Fig. 3(a), (b), and (c), which shows the measured patterns of the cavity backed slot antennas with one strip, five strips, and 30 strips, respectively. It is seen that the antenna patterns are similar to each other and to that of the ordinary CBS antenna. Furthermore, in all the measured results, low levels of cross-polarized radiation, at bore-sight, are observed. The cross-polarized radiation is mostly caused by the close proximity of the coaxial cable that feeds the antenna to the main radiator. The values of the FTBR of the antennas are extracted from the measured radiation patterns and are presented 


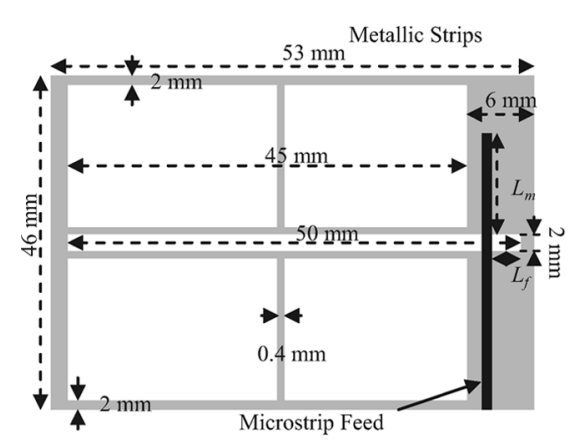

(a)

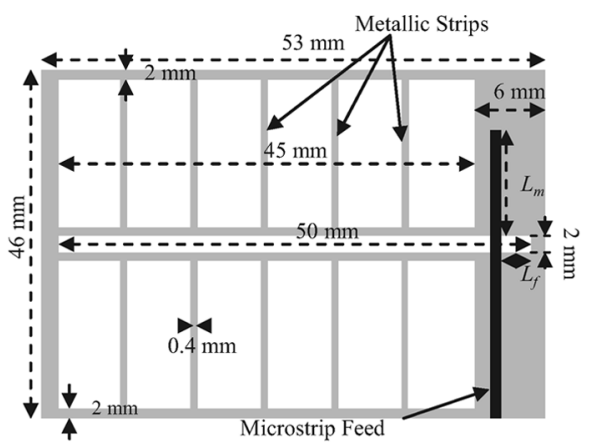

(b)

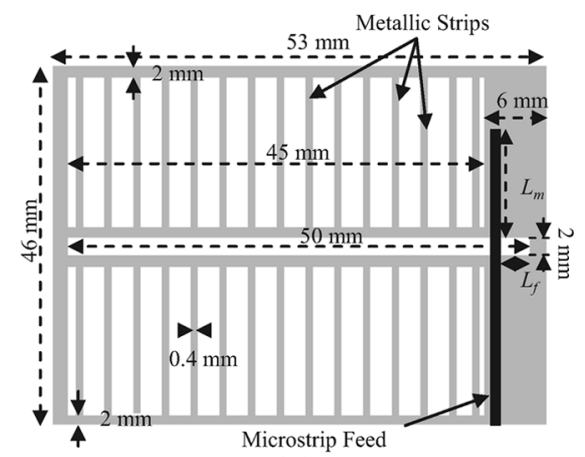

(c)

Fig. 1. Topology of the modified ground plane of the microstrip-fed CBSA. The continuous ground plane is replaced with metallic strips normal to the slot antenna. (a) Modified CBSA with one strip. (b) Modified CBSA with five strips. (c) Modified CBSA with 30 strips.

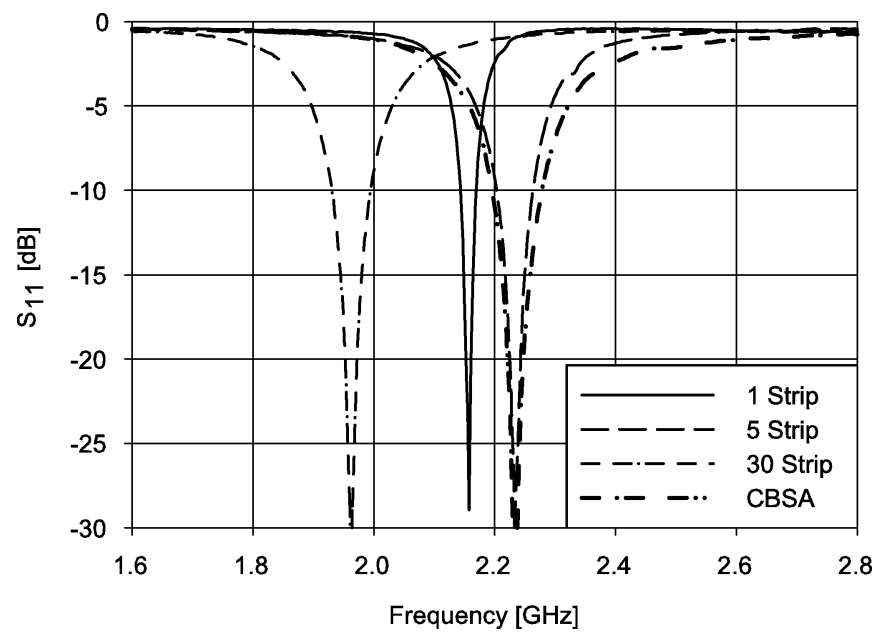

Fig. 2. Measured $S_{11}$ response of the ordinary CBSA and CBSAs with modified ground plane (Section II). The topology of the antenna is shown in Fig. 1.

TABLE II

FeEd Network Parameters of CBSAs of SeCtion II

\begin{tabular}{|c||c|c|}
\hline Antenna Type & $L_{m}$ & $L_{f}$ \\
\hline CBSA with 1 Strip & $6.4 \mathrm{~mm}$ & $1.2 \mathrm{~mm}$ \\
\hline CBSA with 5 Strips & $21.7 \mathrm{~mm}$ & $2.9 \mathrm{~mm}$ \\
\hline CBSA with 30 Strips & $20 \mathrm{~mm}$ & $2.9 \mathrm{~mm}$ \\
\hline Ordinary CBSA & $18 \mathrm{~mm}$ & $2.4 \mathrm{~mm}$ \\
\hline
\end{tabular}

in Table III. It is seen that the FTBR of the antennas are not significantly affected by the number of strips. The gains of the antennas are also measured inside the anechoic chamber using a standard double-ridged horn antenna as reference and the results are presented in Table III along with the calculated directivity values obtained from full-wave simulations. Using the measured gain and calculated directivity values, the antenna efficiency is computed and the results are also presented in Table III. As expected, the antenna gain, efficiency, and bandwidth are considerably affected by the number of strips. As the number of strips is increased both the antenna gain and bandwidth increase and approach to the maximum values provided by the ordinary CBS antenna. As aforementioned, increasing the number of paths of normal component of the electric current causes the antenna to radiate more efficiently and, hence, for fixed physical dimensions, increases its gain. Furthermore, since a greater portion of the power delivered to the antenna is radiated, the ratio of stored energy to that of the radiated power (antenna Q) decreases or equivalently its bandwidth (BW) increases [17]. That is why the ordinary CBSA and the CBSA with one strip have, respectively, the maximum and minimum bandwidth values.

\section{Reduced-Size CaVity-Backed Slot AntenNa (RS-CBSA)}

In this section, the cavity backed slot antennas of the previous section will further be modified to reduce their cavity dimensions and, hence, significantly reduce their overall size. The basic idea is to design the strips, of the modified CBSA presented in the previous section, in a compact fashion while maintaining their electrical length. Fig. 4 shows the reduced-size version of the modified CBS antenna with one strip. Here, the strip is turned into a meander line to reduce the cavity width. The antenna is fed with a $50-\Omega$ microstrip line connected to a $75-\Omega$ open-circuited microstrip line that is meandered to conserve space, but has an overall length of $L_{m}$. The location of the microstrip feed $L_{f}$ and the length of the open circuited stub $L_{m}$ are tuned to match the input impedance of the antenna to that of the line impedance. A total of five different RS-CBSA with different parameters are designed, fabricated, and measured. In all of the antennas, the slot antenna and the microstrip feed line are etched on two different sides of a 0.5-mm-thick RO4003 substrate with a dielectric constant of $\epsilon_{r a}=3.4$, loss tangent of $\tan \delta_{a}=0.0027$, and ground plane size of $53 \mathrm{~mm} \times 18 \mathrm{~mm}$. Four of the antennas have cavity depth of $6.35 \mathrm{~mm}$ and one of them has cavity depth of $3.18 \mathrm{~mm}$. Similar to the previous section, the cavities are machined out of 6.35- or 3.18-mm-thick pieces of TMM3 substrate (from Rogers Corp.) and the sidewalls are then metallized to form the cavity. The geometrical parameters of the antennas are given in Table IV and Fig. 4.

The input reflection coefficients of the RS-CBSAs are measured using a calibrated HP8720D vector network analyzer and the measurement results are presented in Fig. 5. It is observed that independent of the number of meander lines, the antennas are very well matched to $50 \Omega$. The co- and cross-polarized 


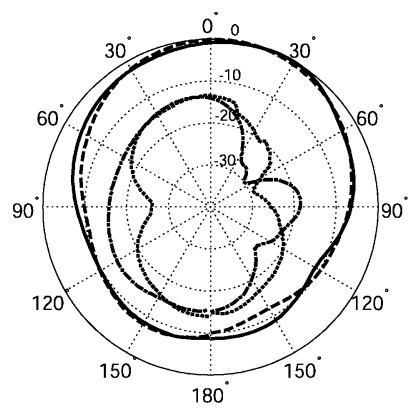

(a)

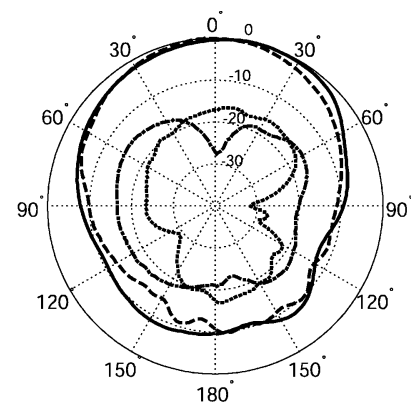

(b)

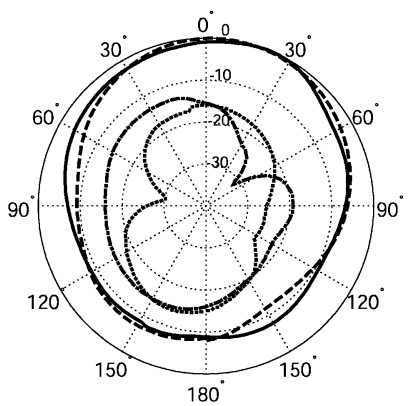

(c)

Fig. 3. Measured radiation patterns of the CBSAs with modified ground plane studied in Section II. (a) CBSA with 1 strip. (b) CBSA with 5 strips. (c) CBSA with 30 strips. Solid line: E-plane co-pol. Dash-dotted line: E-plane cross-pol. Dash-dashed line: H-plane co-pol. Dotted line: H-plane cross-pol.

TABLE III

RADIATION PARAMETERS OF CBSAS OF SECTION II

\begin{tabular}{|c||c|c|c|c|c|}
\hline Antenna Type & BW (\%) & Gain (dBi) & Directivity (dBi) & $\eta_{\text {rad }}$ & FTBR (dB) \\
\hline 1 Strip CBS & 1.2 & 3.4 & 5.8 & $58 \%$ & 8.0 \\
\hline 5 Strips CBS & 2.9 & 4.1 & 5.8 & $68 \%$ & 9.0 \\
\hline 30 Strips CBS & 3.4 & 5.5 & 5.8 & $93 \%$ & 7.9 \\
\hline Ordinary CBS & 4.0 & 5.5 & 5.8 & $93 \%$ & 8.6 \\
\hline
\end{tabular}

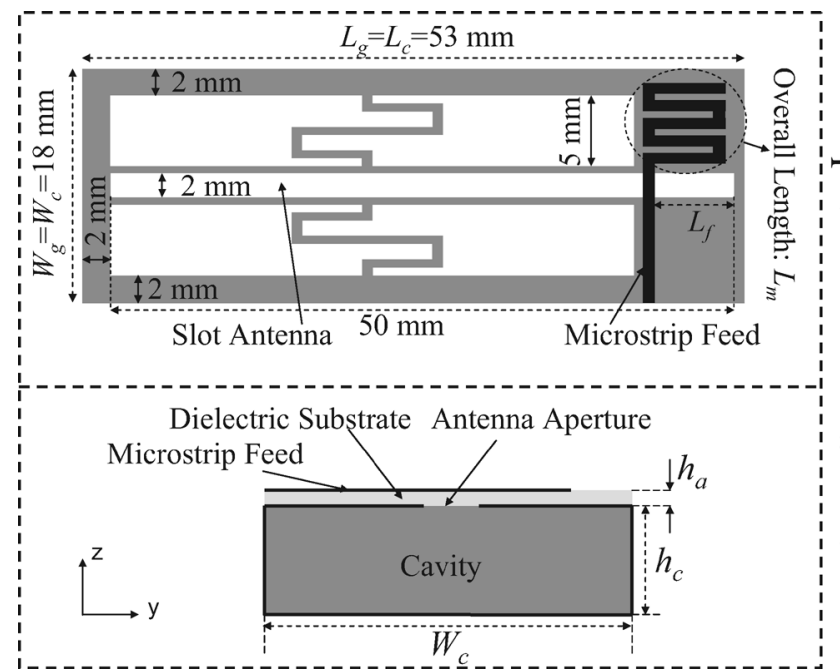

Fig. 4. Topology of the reduced-size-cavity backed slot antenna. The metallic strip is modified such that it maintains its electrical length. The microstrip feed is also meandered to conserve space.

\section{TABLE IV}

Feed Network ANd Physical Dimensions of Miniaturized CBSAS OF SECTION III

\begin{tabular}{|c|c|c|c|}
\hline Antenna Type & $L_{m}(\mathrm{~mm})$ & $L_{f}(\mathrm{~mm})$ & $L_{c} \times W_{c} \times h_{c}\left(\mathrm{~mm}^{3}\right)$ \\
\hline 1 Meander & 43.0 & 5.3 & $53 \times 18 \times 3.18$ \\
\hline 1 Meander & 43.0 & 5.4 & $53 \times 18 \times 6.35$ \\
\hline 2 Meanders & 52.0 & 6.9 & $53 \times 18 \times 6.35$ \\
\hline 3 Meanders & 52.0 & 6.9 & $53 \times 18 \times 6.35$ \\
\hline 4 Meanders & 52.0 & 6.9 & $53 \times 18 \times 6.35$ \\
\hline
\end{tabular}

radiation patterns of the antennas, $\left(\left|E_{\theta}\right|^{2}\right.$ and $\left.\left|E_{\phi}\right|^{2}\right)$, are measured in the E- and H- planes $(y-z$ and $x-z$ planes, respectively). As an example, the normalized radiation patterns of the antenna with two meandered lines and cavity depth of

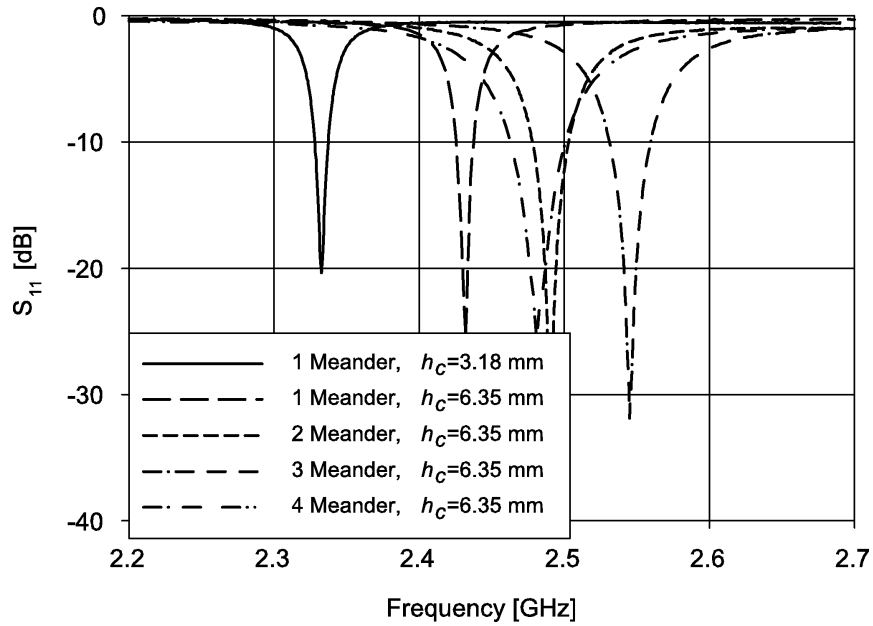

Fig. 5. Measured $S_{11}$ of the miniaturized CBSAs of Section III. The antennas' topology is shown in Fig. 4 and their feed network parameters are given in Table IV.

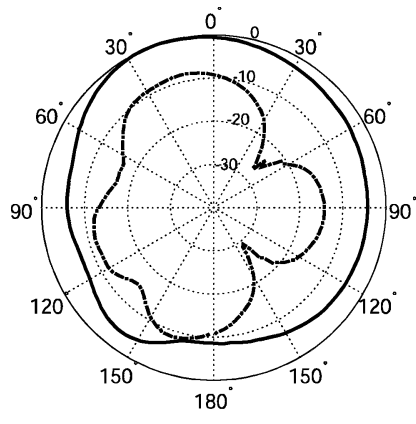

(a)

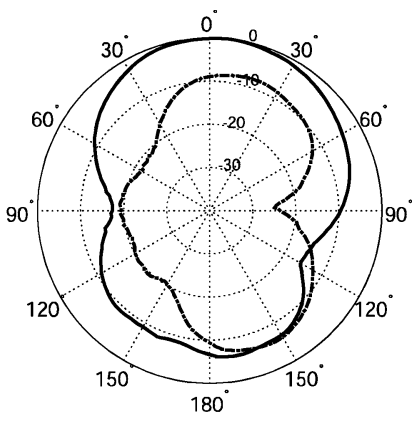

(b)
Fig. 6. Measured radiation patterns of the reduced-size-cavity backed slot antenna that has a ground plane with two meander lines in the (a) E-plane and (b) H-plane. Solid line: E-plane, co-pol. Dash-dotted line: E-plane, cross-pol. Dash-dashed line: H-plane, co-pol. dotted line: H-plane, cross-pol.

$6.35 \mathrm{~mm}$ are shown in Fig. 6. As is observed from this figure, the cross-polarized radiation levels are small at bore-sight. As 
TABLE V

RADIATION PARAMETERS OF THE MINIATURIZED CBSAS OF SECTION III

\begin{tabular}{|c||c|c|c|c|c|}
\hline Antenna Type & BW (\%) & Gain (dBi) & Directivity (dBi) & $\eta_{\text {rad }}$ & FTBR (dB) \\
\hline 1 Meander, $h_{c}=3.18 \mathrm{~mm}$ & 0.4 & -2.3 & 4.0 & $23 \%$ & 6.1 \\
\hline 1 Meander, $h_{c}=6.35 \mathrm{~mm}$ & 0.5 & -0.7 & 3.7 & $36 \%$ & 6.0 \\
\hline 2 Meander, $h_{c}=6.35 \mathrm{~mm}$ & 1.0 & 1.2 & 3.7 & $56 \%$ & 7.5 \\
\hline 3 Meander, $h_{c}=6.35 \mathrm{~mm}$ & 1.2 & 2.85 & 3.7 & $82 \%$ & 7.4 \\
\hline 4 Meander, $h_{c}=6.35 \mathrm{~mm}$ & 1.6 & 3.5 & 3.7 & $95 \%$ & 7.0 \\
\hline
\end{tabular}

in the previous case, the major contributor to the cross-polarized radiation is the close proximity of the coaxial cable feeding the antenna to the main radiator.

The maximum gains of the RS-CBSAs are measured in the anechoic chamber using a standard double-ridged horn antenna as a reference and the results are presented Table V. Moreover, the directivity of the antennas are calculated using full-wave simulations in IE3D [20] and using the measured gain and computed directivity values, the efficiency of the antennas are calculated and the results are presented in Table V. It is observed that as the number of meander lines and the cavity depth increase, the gain and efficiency of the antennas increase as well. The FTBR of the antennas are extracted from the measured radiation patterns and the results are presented in Table V. The FTBRs of the RS-CBSAs are slightly smaller than the CBSAs studied in Section II. This is caused by the significant decrease in the width of the antennas compared to the CBSAs of Section II. Specifically, the total occupied volumes of the miniaturized CBSAs have been reduced by $65 \%$ compared to the CBSAs presented in the previous section.

\section{CONCLUSION}

A new technique for reducing the dimensions of a cavity backed slot antenna is presented. The method is based on replacing the metallic plane around slot antennas with a series of ground plane backed, parallel metallic strips and designing these strips in a compact fashion. It is shown that using this technique, the overall occupied volume of a modified cavity backed slot antenna can be reduced by more than $65 \%$. Furthermore, despite its reduced physical dimensions, the antenna can present a FTBR as high as $7 \mathrm{~dB}$ with relatively low levels of cross-polarized radiation, excellent impedance match, and high radiation efficiency.

\section{REFERENCES}

[1] R. Azadegan and K. Sarabandi, "A compact planar folded-dipole antenna for wireless applications," IEEE Int. Symp. Antennas Propag., vol. 4, pp. 439-442, Jun., 22-27 2003.

[2] N. Behdad and K. Sarabandi, "Bandwidth enhancement and further size reduction of a class of miniaturized slot antennas," IEEE Trans. Antennas Propag., vol. 52, pp. 1928-1935, 2004.

[3] K. Sarabandi and R. Azadegan, "Design of an efficient miniaturized UHF planar antenna," IEEE Trans. Antennas Propag., vol. 51, pp. 1270-1276, Jun. 2003.

[4] R. Azadegan and K. Sarabandi, "A novel approach for miniaturization of slot antennas," IEEE Trans. Antennas Propag., vol. 51, pp. 421-429, Mar. 2003.
[5] J. Galejs, Antennas in Inhomogeneous Media, 1st ed. New York: Pergamon, 1969, ch. 7, pp. 84-103.

[6] _ - "Admittance of a rectangular slot which is backed by a rectangular cavity," IEEE Trans. Antennas Propag., vol. 21, pp. 119-126, Mar. 1963.

[7] C. Cockrell, "The input admittance of the rectangular cavity-backed slot antenna," IEEE Trans. Antennas Propag., vol. 24, pp. 288-294, May 1976.

[8] S. Long, "A mathematical model for the impedance of the cavity-backed slot antenna," IEEE Trans. Antennas Propag., vol. 25, pp. 829-833, Nov. 1977.

[9] — - "Experimental study of the impedance of cavity-backed slot antennas," IEEE Trans. Antennas Propag., vol. 23, pp. 1-7, Jan. 1975.

[10] A. Hadidi and M. Hamid, "Aperture field and circuit parameters of cavity-backed slot radiator," Proc. Inst. Elect. Eng. Antennas, Microw. Propag., vol. 136, pp. 139-146, Apr. 1989.

[11] A. T. Adams, "Flush mounted rectangular cavity slot antennas-theory and design," IEEE Trans. Antennas Propag., vol. 15, pp. 342-351, May 1967.

[12] H. P. Moyer and R. A. York, "Active cavity-backed slot antenna using MESFET's," IEEE Microw. Guided Wave Lett., vol. 3, pp. 95-97, Apr. 1993.

[13] L. Quan and S. Zhongxiang, "An inverted microstrip-fed cavity-backed slot antenna for circular polarization," IEEE Antennas Wireless Propag. Lett., vol. 1, pp. 190-192, 2002.

[14] J. Hirokawa, H. Arai, and N. Goto, "Cavity-backed wide slot antenna," Proc. Inst. Elect. Eng. Antennas, Microw., Propag., vol. 136, pp. 29-33, Feb. 1989.

[15] A. Roederer, "A log-periodic cavity-backed slot array," IEEE Trans. Antennas Propag., vol. 16, pp. 756-758, Nov. 1968.

[16] M. R. Crews and G. A. Thiele Senior, "On the design of shallow depth T-bar fed slot antennas," IEEE Trans. Antennas Propag., vol. 25, pp. 833-836, Nov. 1977.

[17] L. J. Chu, "Physical limitations on omni-directional antennas," J. Appl. Phys., vol. 19, pp. 1163-1175, Dec. 1948.

[18] T. Hikage, M. Omiya, and K. Itoh, "Considerations on performance evaluation of cavity-backed slot antenna using the FDTD technique," IEEE Trans. Antennas Propag., vol. 49, pp. 1712-1717, Dec. 2001.

[19] M. Omiya, T. Hikage, N. Ohno, K. Horiguchi, and K. Itoh, "Design of cavity-backed slot antennas using the finite-difference time-domain technique," IEEE Trans. Antennas Propag., vol. 46, pp. 1853-1858, Dec. 1998.

[20] IE3D Electromagnetic Simulation and Optimization Software, Zeland Software, Inc.

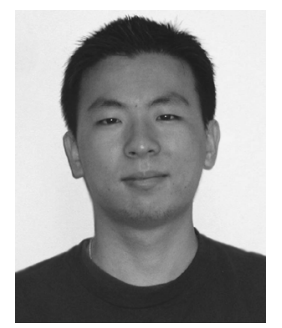

Wonbin Hong ( $\left.\mathrm{S}^{\prime} 05\right)$ received the B.S. degree in electrical engineering from Purdue University, West Lafayette, IN, in 2004. He received the M.S. degree, also in electrical engineering, from The University of Michigan, Ann Arbor, in 2005.

$\mathrm{He}$ is currently pursuing the Ph.D. degree in applied electromagnetics as a Graduate Research Assistant with the Radiation Laboratory, The University of Michigan. His research concentration is on efficiency enhancement and miniaturization of printed antennas. 


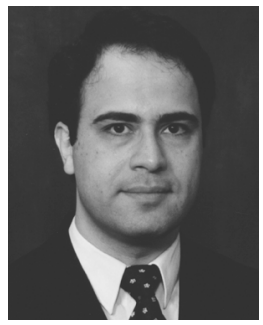

Nader Behdad (S'98) received the B.S. degree in electrical engineering from Sharif University of Technology, Tehran, Iran, in September 2000 and the M.S. degree in electrical engineering from The University of Michigan, Ann Arbor, in 2003.

$\mathrm{He}$ is currently working toward the Ph.D. degree with the Department of Electrical Engineering and Computer Science, The University of Michigan, Ann Arbor. From 2000 to 2001, he worked as a Design Engineer for the Electronics Research Center, Sharif University of Technology. Since January 2002, he has been working as a Research Assistant at the Center for Wireless Integrated Micro-Systems of the University of Michigan.

Mr. Behdad received the Best Student Paper Award in the Antenna Applications Symposium held in Monticelo, IL, in September 2003. He is the second prize winner in the Student Paper competition of the USNC/URSI National Radio Science Meeting in Boulder, CO, in January 2004; and the recipient of the 2005 Horace H. Rackham Predoctoral Fellowship from the Rackham School of Graduate Studies of The University of Michigan.

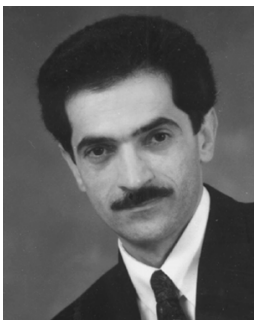

Kamal Sarabandi (S'87-M'90-SM'92-F'00) received the B.S. degree in electrical engineering from Sharif University of Technology, Tehran, Iran, in 1980, the M.S. degree in electrical engineering and mathematics and the Ph.D. degree in electrical engineering both from The University of Michigan-Ann Arbor, in 1986 and 1989, respectively.

$\mathrm{He}$ is Director of the Radiation Laboratory and a Professor with the Department of Electrical Engineering and Computer Science, The University of Michigan. His research areas of interest include microwave and millimeter-wave radar remote sensing, meta-materials, electromagnetic wave propagation, and antenna miniaturization. He has 20 years of experience with wave propagation in random media, communication channel modeling, microwave sensors, and radar systems, and is leading a large research group including two research scientists, ten Ph.D. and two M.S. degree students. Over the past 10 years, he has graduated $22 \mathrm{Ph} . \mathrm{D}$. degree students. He has served as the Principal Investigator on many projects sponsored by NASA, JPL, ARO, ONR, ARL, NSF, DARPA and numerous industries. He has published many book chapters and more than 130 papers in refereed journals on electromagnetic scattering, random media modeling, wave propagation, antennas, meta-materials, microwave measurement techniques, radar calibration, inverse scattering problems, and microwave sensors. He has also had more than 320 papers and invited presentations in many national and international conferences and symposia on similar subjects.

Dr. Sarabandi is a Member of the International Scientific Radio Union (URSI) Commission F and of The Electromagnetic Academy. He received the Henry Russel Award from the Regent of The University of Michigan-Ann Arbor (the highest honor the University of Michigan bestows on a faculty member at the assistant or associate level). He received a 1996 Teaching Excellence Award from the Department of Electrical Engineering and Computer Science and a 1999 GAAC Distinguished Lecturer Award from the German Federal Ministry for Education, Science, and Technology, given to about ten individuals worldwide in all areas of engineering, science, medicine, and law. In 2005, he received the IEEE GRS Distinguished Achievement Award. He also received the 2003/2004 College of Engineering Research Excellence Award and the 2005 Faculty Recognition Award, both from The University of Michigan-Ann Arbor. In the past several years, joint papers presented by his students at a number of symposia (IEEE APS'95,'97,'00,'01,'03,'05 IEEE IGARSS'99,'02, IEEE MTTS'01) have received student prize paper awards. $\mathrm{He}$ is a Vice President of the IEEE Geoscience and Remote Sensing Society (GRSS), a past Chairman of the Awards Committee of the IEEE GRSS from 1998 to 2002, and a Member of the IEEE Technical Activities Board Awards Committee from 2000 to 2002. He was an Associate Editor of the IEEE Transactions on antennas and Propagation and the IEEE Sensors JOURNAL. He is listed in American Men \& Women of Science, Who's Who in America, and Who's Who in Electromagnetics. 